\title{
The Experimental Study on Computer Video Feedback in Tennis Serve Teaching Effect
}

\author{
Zhenkun Zheng \\ Shanghai University of Engineering Science P.E Department, Shanghai, 201620 \\ Zhengzhenkun61@sina.com
}

\begin{abstract}
Through the experimental comparison of $33 \%$ computer video feedback and $33 \%$ language feedback in the effect of tennis serve teaching, we found that $33 \%$ computer video feedback group was better than $33 \%$ language feedback group and the control group without feedback.. The analysis shows that computer video feedback has the advantage of improving learners' learning interest, independent learning ability, making up for the deficiency of teachers' demonstration, which is worth promoting in the teaching .
\end{abstract}

Keywords- computer; video; feedback; tennis; effect

\section{PREFACE}

Tennis is getting increasingly popular among the majority of the students in colleges and universities, with its unique charm to attract more and more students to go to the tennis court. For beginners, the overhand serve is a bit more difficult to master in numerous tennis technology. The author engaged in the university tennis teaching and training for ten years and found that for most beginners , to master the overhand serve technology is not very desirable within the limited class hour . Investigation of other college tennis teacher also shows that. Therefore, to explore a better, more excellent tennis teaching method, teaching means is of great significance.

Feedback refers to provide the practitioners with the relevant information of skills in the process of practice or after the practice. In the learning process of motor skills learning and control, feedback is essential. Adams said: without feedback, there is no learning. A lot of studies have shown that: the feedback of appropriate frequency and time is essential for practitioners to master sports skills, not only can promote the movement target, but also encourage practitioners to have continuous efforts for the target . The language feedback of traditional sports skill teaching has been shown to be effective, of course, which also has its shortcomings, such as attach importance to the teachers' management while neglecting students' active learning. Now, the computer technology has been very widely used in the field of education, with the help of sonographic features of the modern media, and combining the reasonable elements of traditional teaching to transmit the teaching information and feedback to adjust whether can be accepted by students, whether can achieve better teaching effect, is the target of this study.

\section{THE OBJECT OF STUDY AND EXPERIMENTAL METHODS}

\section{A. The object of study}

24 freshman boys of the special elective tennis for the first time, without tennis practice experience, were randomly divided into 3 groups, each group of 8 people.

\section{B. Experimental methods}

\section{1)Experimental equipment}

Equipped with the Premiere pro1.5 editing software of Dell Ins15r-2528 notebook, the Sony HDR-Pj660E digital camera .

\section{2)Experimental operation}

The 3 groups were randomly divided into separately overhand serve teaching. The teaching process is the teacher's introduction, explanation , demonstration, students' exercise without balls, complete movement practice with balls, net practice in pairs. Teaching process is accomplished by the same teacher, students do not know the experiment purpose, methods, and ensure the skill practice only during the experiment.

Group 1 for video feedback group, after three times of each exercise to give a time of the 90 s video feedback (including clips from 60s practice action and 30s standard action), is $33 \%$ video feedback. The location is located in $6 \mathrm{~m}$ of the left front and right front of the practitioners in turn. Group 2 is a language feedback group, after three times of each exercise to give an evaluation, namely as $33 \%$ language feedback. Group 3 for the control group, after three practice not to give any evaluation, namely the $0 \%$ feedback.

For 8 weeks, 2 lessons per week, $90 \mathrm{~min}$, during the ninth week of class, the unified evaluation by a non experimental teacher, evaluation consists of two parts ,the standards and technical evaluation, each accounted for $50 \%$, the standard part requires every student to complete 20 overhand serve with $70 \sim 80 \%$ of maximum strength (each area 10 times, first right then left for rotation), 5 points of one success, full mark is 100 points; technical assessment were graded according to finish out the standard action, full mark is also 100. After the experiment, the data using SPSS19.0 statistical software for the Independent Samples T Test. (Note: the feedback frequency is an important variable in the motor skill learning but additional feedback will lead to practitioners to ignore their inner feedback, for additional feedback will 
produce dependence, hinder the development of the error detection and correction mechanism .Therefore, frequently provided feedback is not conducive to master the skills. Access to a large number of studies have found that: $33 \%$ of frequency feedback on Taijiquan, football, basketball, tennis and other sports learning has a significant effect, so the study is on 33\% of frequency feedback, as for the effects of other frequency feedback for tennis skills need to be further discussed in the future.)

\section{THE RESULTS OF THE STUDY}

In the experiment teaching of 8 weeks, 3 groups of students keep a good attendance. The evaluation score of the ninth week was used for statistical processing by the Independent Samples T Test.(table 1)

TABLE1 THE COMPARISON TABLE OF THE EVALUATION SCORE OF 3 EXPERIMENTAL GROUPS

\begin{tabular}{ccccc}
\hline & $\begin{array}{c}\text { The } \\
\text { number }\end{array}$ & $\mathrm{X} \pm \mathrm{S}$ & $\begin{array}{c}\text { the } \\
\text { highest } \\
\text { score }\end{array}$ & $\begin{array}{c}\text { The } \\
\text { lowest } \\
\text { score }\end{array}$ \\
\hline $\begin{array}{c}33 \% \text { computer video } \\
\text { feedback group }\end{array}$ & 8 & $67.50 \pm 6.41$ & 77.5 & 57.5 \\
$\begin{array}{c}33 \% \text { language feedback } \\
\text { group }\end{array}$ & 8 & $60.31 \pm 6.87$ & 72.5 & 52.5 \\
The control group & 8 & $47.19 \pm 8.50$ & 57.5 & 35 \\
\hline
\end{tabular}

In the experiment teaching of 8 weeks, 3 groups of students keep a good attendance. From table 1, the evaluation score of 33\% language feedback group is the highest, on average , 7.19 points higher than 33\% language feedback group, 20.31 points higher than control group, while the evaluation score of the control group nearly without feedback was significantly behind the two experimental group with feedback, respectively 13.12 and 20.31 points behind.

TABLE 2 THE ANALYSIS AND COMPARISON OF THE INDEPENDENT SAMPLES T TEST TABLE OF THE EVALUATION SCORE OF 3 GROUPS OF PRACTITIONERS

\begin{tabular}{cccc}
\hline Group & $\begin{array}{c}\text { 33\% computer } \\
\text { video feedback } \\
\text { group }\end{array}$ & $\begin{array}{c}\text { 33\% computer } \\
\text { video feedback } \\
\text { group }\end{array}$ & $\begin{array}{l}33 \% \text { language } \\
\text { feedback } \\
\text { group }\end{array}$ \\
\cline { 2 - 4 } & $\begin{array}{c}\text { 33\% language } \\
\text { feedback group }\end{array}$ & The control group & $\begin{array}{c}\text { The control } \\
\text { group }\end{array}$ \\
$\mathrm{P} \quad \mathrm{P}=0.048<0.05$ & $\mathrm{P}=0.000<0.01$ & $\begin{array}{c}\mathrm{P}=0.004< \\
0.01\end{array}$
\end{tabular}

From table 2, the evaluation scores of 33\% computer video feedback group and 33\% language feedback group compared with the control group had significant difference. The feedback from the computer and language feedback group also had significant difference $(\mathrm{P}=0.048<$ $0.05)$. The test results showed that: the teaching effect of computer video feedback experimental group was much better than language feedback group and the experimental group without feedback. In experiment teaching, teachers also felt that the overall serve action of computer video feedback group was more concise, with less unnecessary redundant movement as well as better driving stability and velocity than the other two groups.

\section{DISCUSSION AND ANALYSIS}

A. Analysis of the differences between the 33\% frequency language feedback group and the control group.

Through the tests, it clearly showed that the mastery of the $33 \%$ frequency feedback group was obviously superior to the control group on the overhand serve technology. Experimental results also proved once again that the feedback was the indispensable in the process of learners learning motor skills. Tennis serve action requires that the head, arm, waist and hip and leg movements of the practitioners can be coordinated. The practitioners must master the standard serve action, must have a clear concept of action and form the right sports image, in order to compare their completed action with the standard action. But for beginners, to produce the wrong, superfluous action is inevitable, in this process, if they can not get feedback from the teacher, their right action will not be enhanced. The wrong action will not be corrected in time. While they practice, the practitioners found that they sent the balls under the net or out of bounds again and again. Their mood was very easy to become irritable or anxious and they lost self-confidence little by little which will affect the practitioners' learning enthusiasm and initiative.

\section{B. Analysis of the differences between the 33\% computer video feedback group and 33\% language feedback group}

The results of Table 2 show that after 8 weeks of the overhand serve teaching, the implementation of 33\% computer video feedback control group contrast to 33\% language feedback group, has made the better teaching effect.

A large number of domestic and foreign experimental research shows, the appropriate oral or language feedback for practitioners is essential and effective to learn and master the sports skills. With the development of educational technology, some other forms of feedback have been used in the teaching practice, such as video feedback, model feedback, visual feedback, biological feedback. For the study of the feedback, conclusions are not uniform. Many scholars believe that, as long as they master the appropriate frequency and time of the feedback, video, visual feedback is helpful for the learners to learn motor skills. Of course, some scholars have different conclusions through the experiment, Rothstein\& Arnold (1976) pointed out that the visual feedback and verbal feedback effect on the motor skill learning in $63 \%$ of literature showed that: the visual feedback for motor skill learning did not produce more positive effect than other feedback ways [1]. Similarly, the study of Rikli \& Smith (1980) and Emmen (1985) on tennis serve based on the visual feedback showed that the effect of the visual feedback for the learning of motor skills did not produce significant effect[2].

Computer video feedback inherited the reasonable 
elements of traditional teaching, using audio-visual function of the modern media mainly on computer to display practitioners kinematics characteristics of the operating action, by suggesting the practitioners movement characteristics associated with exercise result, to enhance the intrinsic sensory feedback. Language feedback is effective, but also has its limitations. In the process of teaching sports skills, with too much attention to teachers' leading role, teachers carry on the explanation, demonstration in class while students continue to imitate the practice. Most practitioners are in a passive learning state and limit the development of their cognition and cognitive ability. In the process of teaching sports skills, not only to let students learn, but also let the students know how to learn well, computer video feedback compared with traditional language feedback, has the following advantages:

Firstly, to improve initiative learning of the practitioners. Computer video feedback is applied less in sports technique teaching. In the teaching process, first of all ,students have a lot of curiosity, facing the camera with enthusiasm to cooperate. Through the feedback of 90s video, the students have an intuitive, clear understanding on their completed action, know their own mistakes and shortcomings of each action. After watching the video, many students will communicate with teachers actively as well as discuss grip, toss, back swing together. This phenomenon is very rare in the language feedback group and control group.

Secondly, to promote autonomous learning ability of the practitioners. Scholar Pang Weiguo summarized the self-regulated learning as: based on the students with intrinsic motivation of "want to learn"; based on the students mastering the learning strategies of "will learn"; based on the will to try to "keep on learning" [3]. At the primary stage of learning the overhand serve, it is a very common thing for practitioners to have or repeat wrong, superfluous action, because at this time they have not got very clear concept of correct action. They know they are wrong but don't know what is wrong ,at this time they can only follow the teachers' language feedback to correct. Through the Feedback of the computer video, practitioners can clearly see their completed action. To contrast the standard action video, it is easy to find the deficiency of their movements, inspire them to complete the action accurately. When students find their own mistakes, combined with the teachers' evaluation on their own completed actions, they will observe, think, communicate, analysis to complete the action constantly close to the standard action model. When the trainees can see their progress, their changes, their training passion is not easy to decline. In the teaching process, the impatience which often appears on the face of the students because of repeated practice is rare. The sluggish phenomenon owing to the dull practice is gone. The teaching process changed teacher-centered to student-centered.
Thirdly, to make up for the inadequacy of the teachers' demonstration. Tennis is a very technical sport, especially the serve technology for beginners, demonstration plays an important role. The study of Richard Maizher(1992)shows that visual demonstration can promote beginners to accept the correct mode, visual information of skill demonstration enable beginners to easily obtain coordination of the limbs movement ${ }^{[4]}$. The impression of demonstration action in the class for the beginners for the first time in his mind influences the next learning. At present, tennis teachers have uneven level, not every demonstration of the teacher can be successful. At this time, using computer video feedback can make up for the inadequacy of teachers' demonstration action. Through the playback, slow down, parking of the normative action model, we can display complete action at any time, along with the advancement of learning, but also show practitioners or introduce them the famous professional tennis players' serve to improve their understanding of serve technique.

\section{V.CONCLUSIONS AND RECOMMENDATIONS}

The results of the study showed that in 8 weeks of tennis ball teaching 33\% computer video feedback group is better than the 33\% language feedback group and the control group, which tells us 33\% frequency of computer video feedback is effective for beginners to learn and master the tennis movement technology.

The analysis showed that the implementation of computer video feedback can stimulate learners' interest in learning, improve self-learning ability, make up for the inadequacy of the teachers' class demonstration, which is worth to promote in the teaching practice.

This paper only discusses the teaching effect of using $33 \%$ computer video feedback for beginners after 8 weeks of learning tennis overhand serve. For a higher level of practitioners, whether more time to practice, different sports and different feedback frequency can produce obvious teaching effect, need to be further studied.

\section{REFERENCES}

[1] Rothstein\& Arnold (1976). Bridging the gap: application of research on videotape feedback and bowling. Motor Skill: Theory into Practice1, 36 - 62

[2] Rikli \& Smith (1980). Videotape feedback effects on tennis serving form.Perceptual and Motor Skills 50895 - 901

[3] Pang Weiguo. Primary and middle school students' autonomous learning teaching mode of [D], Shanghai: East China Normal University doctoral thesis.25-32

[4] Richard, Mai djer. Journal of visual demonstration and limbs international sports [J]. coordinated motion research in psychology, 1992 (23) 3:207-226

[5] Tan Jiahui, PhD. Focus of [D]. research of additional experimental papers pay attention to soccer motor skill learning. Beijing: Beijing Sport University, 2012Xue Yu of computer video feedback advantage in sports technique teaching [J]. Inner Mongolia sports science and technology, 2012 (4): 37-39 